\title{
APLICAÇÃO MÓVEL ESPECÍFICA PARA SMARTPHONES A FIM DE SUPORTAR AS INTERAÇÕES DOS ESTUDANTES COM O SISTEMA DE AVALIAÇÃO DA APRENDIZAGEM (SAA) BASEADO EM APIS E WEBSERVICES.
}

\author{
ITU/SP MAIO/2018
}

\author{
Dilermando Piva Junior - Fatec / CPS - pivajr@gmail.com \\ Andre Ricardo da Costa - Fatec Itu - arc.andrecosta@gmail.com
}

Tipo: Investigação Científica (IC)

Natureza: Planejamento de Pesquisa

Categoria: Métodos e Tecnologias

Setor Educacional: EDUCAÇÃO SUPERIOR

\begin{abstract}
RESUMO
Indicadores de mercado e pesquisas recentes vem mostrando que o não desenvolvimento de aplicações móveis resultam em uma perda significativa de acesso, e, consequentemente, da fatia de mercado. Em recém pesquisa junto aos estudantes que utilizam o Sistema de Avaliação de Aprendizagem (SAA), observou-se que o acesso dos estudantes ao sistema se deu, na sua maioria, por meio dos smartphones. Por ser o SAA um software desenvolvido para o ambiente Web, mesmo com a implementação de recursos que permitem a interoperabilidade e ajustes na exibição para diferentes tipos de dispositivos (responsivo), ocorreram dificuldades na utilização de determinados recursos, em especial, no processo de avaliação. Com vistas a potencializar a utilização do sistema pelos estudantes, o desenvolvimento de uma aplicação móvel para o sistema SAA se faz necessária. Assim, este projeto tem como objetivo desenvolver uma aplicação móvel para smartphones a fim de suportar as interações dos estudantes com o sistema de avaliação da aprendizagem (SAA) baseado em WebServices e APIs. A metodologia proposta passa pela revisão da literatura, estudo de técnicas para construção de APIs e Webservices, análise e escolha de framework para o desenvolvimento da aplicação móvel. Uma vez cumpridas tais etapas, será necessário uma nova modelagem das estruturas centrais do sistema SAA para comportar a implementação da aplicação móvel, via APIs e Webservices. Por fim, uma vez desenvolvida a aplicação, a mesma deverá ser testada em casos reais, junto aos estudantes, com a coleta de informações que indiquem se a implementação atingiu o objetivo de potencializar a utilização do sistema pelos estudantes.
\end{abstract}

Palavras-chave: Webservices, Aplicação Mobile, SAA. 


\section{Introdução}

É possível observar que a prática de avaliação nas Instituições de Ensino Superior está, muitas vezes, centrada no emprego de avaliações somativas como forma básica de "medir" quanto conteúdo foi "aprendido" pelos estudantes (Moraes, 2014).

As razões são diversas e não cabe aqui discuti-las, é certo que a legislação tem contribuído para consolidar esse procedimento, especialmente em cursos desenvolvidos a distância, dado que é exigida avaliação presencial que prepondere sobre as demais formas avaliativas e, com isso se dê o avanço para etapas posteriores do desenvolvimento de um dado curso.

No mundo contemporâneo, a mudança do paradigma educacional do ensino para a aprendizagem tem permitido novas possibilidades de condução dos processos avaliativos, que passam a ter foco no processo, no dia-a-dia, no "feedback" dado aos estudantes para seu desenvolvimento e, não apenas por uma questão de escala, o emprego da tecnologia tem se tornado cada vez mais importante e necessário.

É nesse contexto que se insere o Sistema de Avaliação da Aprendizagem - SAA(Piva Jr et al, 2016), que é uma ferramenta que potencializa a ação docente, tornando possível a aplicação de avaliações formativas ao longo do processo de ensino-aprendizagem, independentemente da metodologia utilizada (presencial ou a distância), de haver ou não a inversão da sala de aula, ou de serem utilizados ou não ambientes de aprendizagem.

Uma das principais vantagens de proporcionar a verificação da aprendizagem por meio de métodos formativos consiste na possibilidade de prover correções de rumo - ou retomada dos mesmos, caso seja verificado um desvio prejudicial aos objetivos do projeto e ao aprendizado dos estudantes, de forma geral.

Com a utilização do sistema SAA, após um planejamento inicial, o professor consegue estabelecer um acompanhamento mais individualizado, mesmo trabalhando com um número grande de estudantes.

A Figura 1(A), a seguir, representa a sequência de planejamento de uma determinada disciplina que deve ser realizada por um docente no SAA. Depois de realizados os cadastros iniciais, deve-se observar as etapas operacionais frequentes do sistema. Essas etapas são descritas na Figura 1(B). Em termos práticos, uma vez que se chega a uma data planejada de aula, o sistema aponta que pode ser realizado o lançamento das 
presenças dos estudantes.
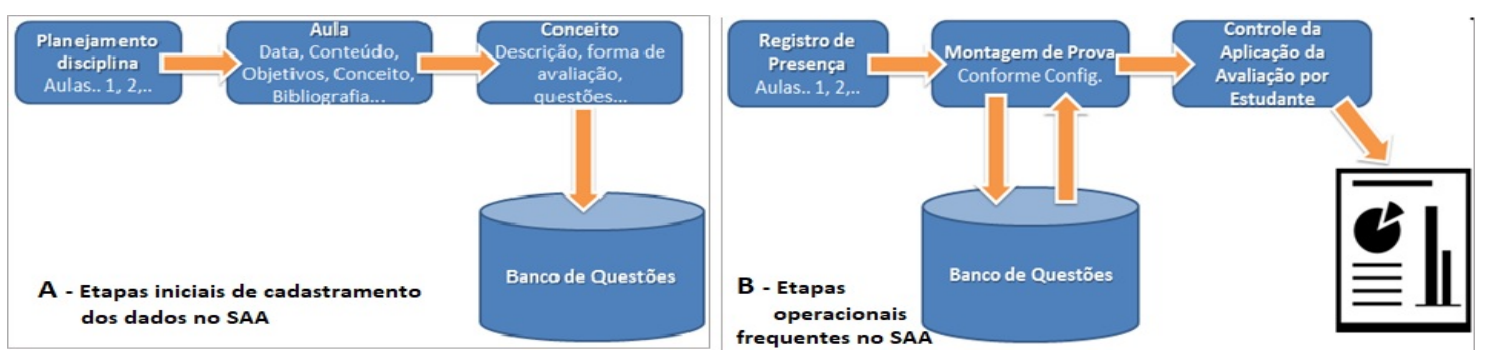

Figura 1 - Etapas realizadas no SAA

Uma vez pontadas as presenças, apenas e tão somente para os alunos que estiveram presentes àquela atividade, o sistema irá montar uma avaliação (individualizada) que será associada aos estudantes que estiveram presentes. Uma mensagem por e-mail será enviada a cada estudante presente para que ele se lembre de que existe uma avaliação a ser realizada, com informações da disciplina, da aula e a quantidade de minutos que o estudante deve reservar (em média) para respondê-la.

Após a realização da avaliação, o sistema disponibilizará uma série de relatórios e gráficos para que o professor, o tutor, o estudante, o coordenador e o diretor da unidade, possam tomar as ações convenientes

O SAA foi desenvolvido e aplicado junto a disciplinas de cursos na área de Computação e Informática, em modelos tradicionais de curso (cursos presenciais). Os testes foram realizados em três Faculdades de Tecnologia do Centro Paula Souza. A primeira etapa envolveu duas turmas de um mesmo professor, num total de 109 estudantes. A segunda etapa, cinco professores em sete turmas/disciplinas diferentes, num total de 387 estudantes. Na primeira etapa, aferiu-se um ganho médio de aprendizagem da ordem de $17,64 \%$ e, na segunda etapa, os resultados mostraram valores com melhoria da ordem de $20 \%$.

Ao longo desses testes, o SAA foi avaliado pelos professores e estudantes. Os resultados dessa avaliação indicaram pontos fortes e fracos da aplicação do sistema, e do próprio sistema. Um dos pontos indicados para melhoria é a forma de acesso no sistema pelos estudantes. A maioria dos estudantes possui e acessa o SAA por seus smartphones. Mesmo o sistema estando preparado para ser executado, por meio de um navegador (browser), em dispositivos móveis, as avaliações indicam a necessidade de construção de uma aplicação móvel específica para smartphones. Não só pela estética, mas principalmente para melhoria da segurança do processo de avaliação e da interface e processo de interação dos estudantes com o sistema. 


\section{Objetivo geral do projeto}

Este projeto tem como objetivo desenvolver uma aplicação móvel para smartphones a fim de suportar as interações dos estudantes com o sistema de avaliação da aprendizagem (SAA) baseado em WebServices.

\section{Objetivos específicos}

- Estudar o funcionamento e estrutura do Sistema de Avaliação da Aprendizagem (SAA) e as estratégias de aplicação de avaliações formativas por meio de aplicações móveis;

- Estudar os processos envolvidos na construção de WebServices e APIs, envolvendo aplicações cliente-servidor e dispositivos móveis.

- Remodelar a estrutura de banco de dados e serviços do Sistema de Avaliação da Aprendizagem (SAA) a fim de suportar a criação de WebServices para acesso remoto (via APIs) de funcionalidades de avaliação e exibição de relatórios e gráficos;

- Desenvolver e testar a aplicação móvel para smartphone, baseado em APIs e WebServices.

\section{Justificativa}

A maioria das grandes empresas possuem as duas opções: web site responsivo e aplicativo móvel (app). Uma das razões para as empresas manterem as duas opções é que ainda existem pessoas resistentes a instalar um aplicativo. Em termos mais técnicos, a utilização de um aplicativo móvel (app) torna o processo mais ágil, seguro e com uma melhor experiência para o usuário. Empresas que vendem produtos pela internet indicam que a taxa de conversão (vendas) é maior devido a usabilidade e performance proporcionada pelo aplicativo.

Assim, em virtude das avaliações realizadas pelos estudantes do sistema SAA e seguindo a tendência do mercado de desenvolvimento de sistemas, a construção de uma aplicação móvel para o sistema SAA especificamente para os estudantes é importante, e tudo indica que, com a melhoria da usabilidade e performance proporcionada pela aplicação móvel, a efetividade das avaliações será maior, possibilitando melhoria no processo de acompanhamento e aprendizagem dos estudantes. 


\section{Referencial teórico}

Segundo El-Kassas et al. (2015), o desenvolvimento de aplicativos móveis diferencia-se do desenvolvimento de outros tipos de software por possuir particularidades e restrições. Os desenvolvedores devem ter em mente aspectos como as capacidades e especificações dos dispositivos móveis, a mobilidade, o design e navegabilidade de interface gráfica, segurança e privacidade do usuário.

De acordo com Corral, Janes e Remencius (2012), aplicações móveis são desenvolvidas dinamicamente e lançadas no mercado, geralmente em pequenos ciclos. Essa forma de distribuição, geralmente resulta em produtos de pequeno porte, que são, na sua grande maioria, comercializados a preços baixos. As equipes de desenvolvimento também tendem a ser pequenas.

Além dessas particularidades, El-Kassas et al. (2015) nos indica quatro outras que devem ser consideradas no processo de desenvolvimento de aplicações móveis:

- Limitações de recursos: As capacidades de processamento e de armazenamento dos dispositivos móveis são limitadas e a conectividade pode ser afetada por ser uma plataforma móvel.

- Ecossistema heterogêneo: O desenvolvimento de aplicações móveis encontra-se em um contexto heterogêneo devido aos diferentes sistemas operacionais e à grande quantidade de dispositivos distintos, com variações de poder computacional, configurações de hardware e tamanhos de tela. Essas singularidades devem ser consideradas no desenvolvimento de apps, o que pode implicar na necessidade de diferentes versões de uma mesma aplicação.

- Experiência de uso: é importante que as aplicações sejam simples e que possuam uma interface amigável para atender às expectativas dos usuários, caso contrário, devido ao grande número de aplicações disponíveis, o app pode ser descartado e substituído por outros presentes nas lojas de aplicativos.

- Manutenção: plataformas mobile passam por constantes atualizações que podem afetar apps já desenvolvidos, a ponto de torná-los inutilizáveis ou causarem desconforto aos usuários. Para que a aplicação continue a funcionar corretamente, são necessárias frequentes manutenções e atualizações. Atualizações de apps desenvolvidos para diferentes plataformas implicam na alteração de cada uma das versões desenvolvidas. 
O desenvolvimento de aplicações móveis se baseia em duas principais abordagens: 0 desenvolvimento nativo e o desenvolvimento multiplataforma. Segundo El-Kassas et al. (2015), aplicações nativas são desenvolvidas com o uso de ferramentas e linguagens específicas, geralmente fornecidas pelos proprietários daquela determinada plataforma, tais como SDK e frameworks específicos. Os aplicativos (apps) ficam vinculados a esse ambiente, executando apenas nos dispositivos daquela determinada plataforma na qual foram construídos. Caso exista a necessidade de desenvolvimento para uma outra plataforma, o mesmo deverá ser feito, praticamente do zero, utilizando para isso, os ambiente de desenvolvimento e linguagens específicas daquela plataforma.

Portanto, para desenvolvimento de uma aplicação nativa deve-se desenvolver o mesmo aplicativo para cada plataforma que se deseja alcançar, o que exige tempo, investimento e uma equipe com conhecimento nas variadas tecnologias utilizadas pelas distintas plataformas. Assim, soluções de desenvolvimento multiplataforma possibilitam a implementação de uma aplicação que pode ser executada em diferentes plataformas.

Atualmente, no mercado existem várias opções de ambientes, linguagens e frameworks para o desenvolvimento multiplataforma. Cada qual com suas características, pontos positivos e negativos. Alguns exemplos são: PhoneGap, Cordova, AngularJS e lonic.

A equipe de desenvolvimento deve decidir para qual plataforma desenvolver, tendo sempre em vista que, ao não criar o aplicativo para uma plataforma, grande parcela do mercado pode estar sendo desconsiderada, o que acaba por reduzir o alcance da aplicação. (CORRAL; JANES; REMENCIUS, 2012).

Para o desenvolvimento de várias aplicações para cada plataforma de maneira nativa (como já mencionado), é preciso passar por todo o processo de desenvolvimento novamente, pois para cada plataforma há uma arquitetura distinta que deve ser seguida, padrões de código e interface de usuário e APIs próprias (HOLZINGER; TREITLER; SLANY, 2012). Isso sugere que, cada aplicação deve ser codificada considerando as diferentes arquiteturas, componentes e guidelines, customizadas o máximo possível para ser a mesma aplicação em plataformas distintas e por fim, gerados novos executáveis e distribuídos nas diferentes lojas de aplicativos (CORRAL; JANES; REMENCIUS, 2012).

Isso implica em planejar cada app com base em uma plataforma distinta, por mais que se trate do mesmo aplicativo, o que acaba por encarecer e tornar mais lento o desenvolvimento (EL-KASSAS et al., 2015). 
No entanto, quando se pensa em desenvolvimento multiplataforma, o projeto fica isolado em relação à plataforma, pois o projeto é feito pensando-se apenas em uma arquitetura, um ambiente de desenvolvimento e um conjunto único de ferramentas e não de acordo com as peculiaridades de cada plataforma. Após o desenvolvimento do núcleo da aplicação, podem ser feitas customizações para adaptar melhor o aplicativo para cada plataforma alvo da distribuição, criando assim um produto final verdadeiramente multiplataforma (CORRAL; JANES; REMENCIUS, 2012).

A Figura 2 apresenta as diferenças existentes nos processos de desenvolvimento de um app utilizando tecnologias nativas e multiplataformas.

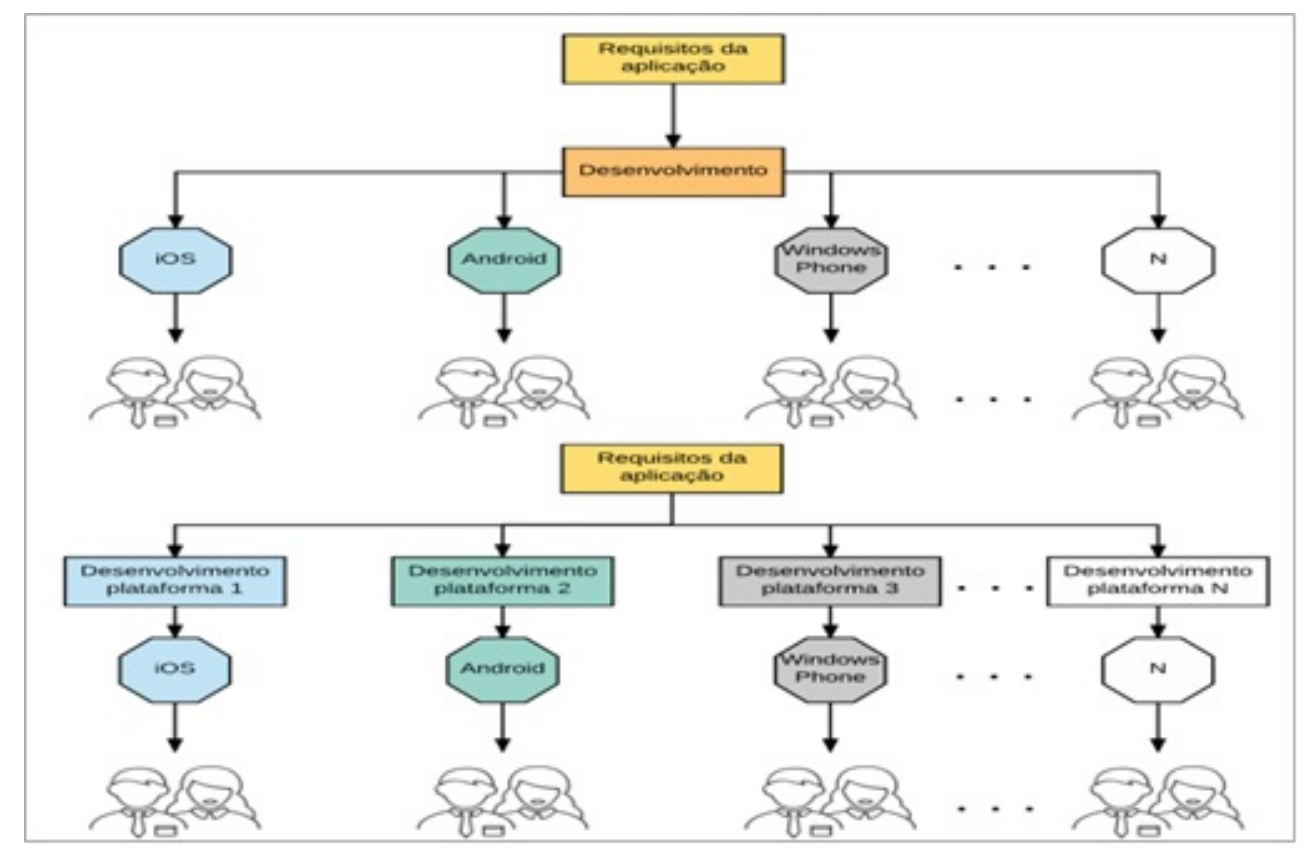

Figura 2 - Desenvolvimento Multiplataforma e Nativo (baseado em Corral, Janes e Remencus, 2012)

Com base nessas características e no trabalho de diversos outros autores, Britto e Silva e Matos (2016), congregaram as vantagens e desvantagens de se desenvolver aplicações móveis utilizando o desenvolvimento nativo ou o multiplataforma. Essas informações estão concentradas na Tabela 1, a seguir.

Tabela 1: Comparativo entre Vantagens e Desvantagens do desenvolvimento Nativo e Multiplataforma. 


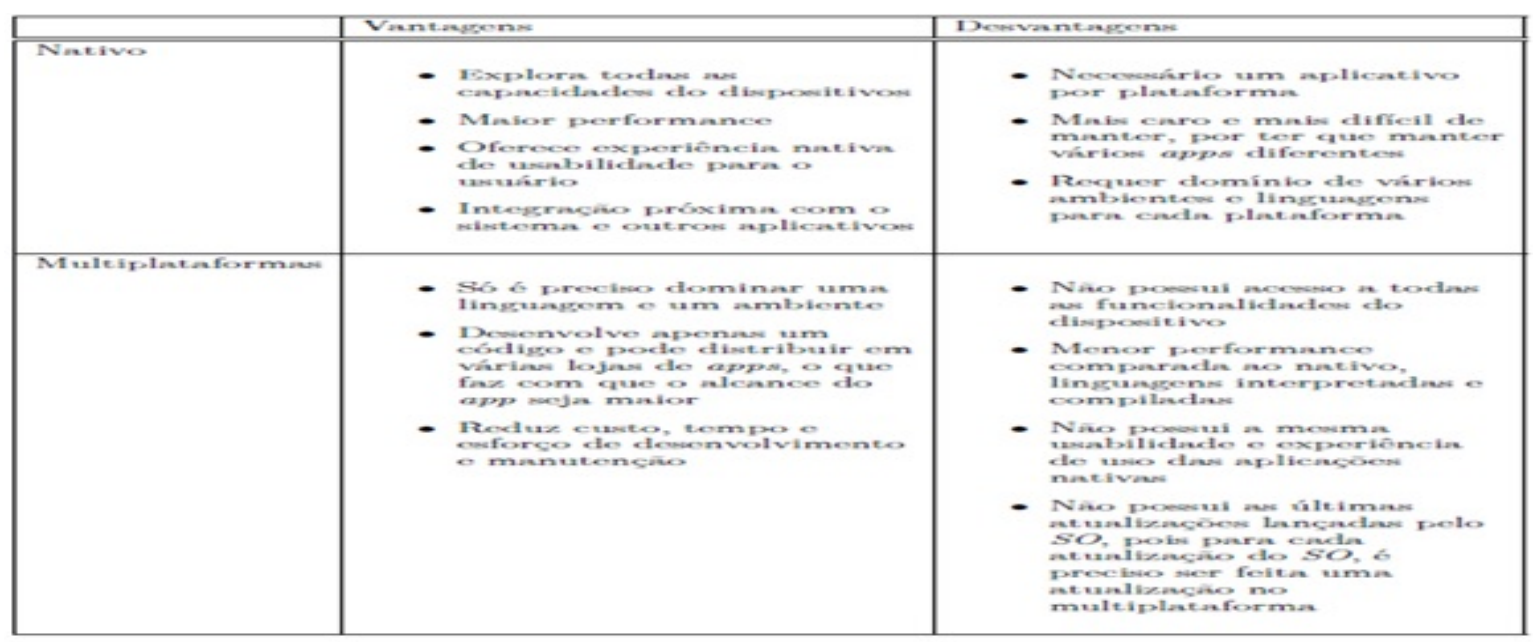

Fonte: (BRITTO E SILVA; MATOS, 2016, p. 39)

Portanto, como a aplicação a ser desenvolvida deve ser feita em pelo menos duas plataformas (Android e IOS), e as desvantagens não trazem impactos significativos para o objetivo principal da aplicação (SAA), a opção por desenvolvimento multiplataforma parece ser a mais indicada.

Ao analisar brevemente suas características, as quais devem ser ao longo da presente pesquisa aprofundados e melhor analisados, o framework que melhor atende aos requisitos necessários para o desenvolvimento da aplicação móvel do SAA é o lonic. Trata-se de um framework livre para o desenvolvimento de aplicativos híbridos utilizando tecnologias web como HTML, CSS e JavaScript otimizadas para dispositivos móveis (DRIFTY, 2016).

Revisando o projeto inicial do Sistema de Avaliação da Aprendizagem, é apresentado na Figura 3, a modelagem completa do sistema, com suas funcionalidades.

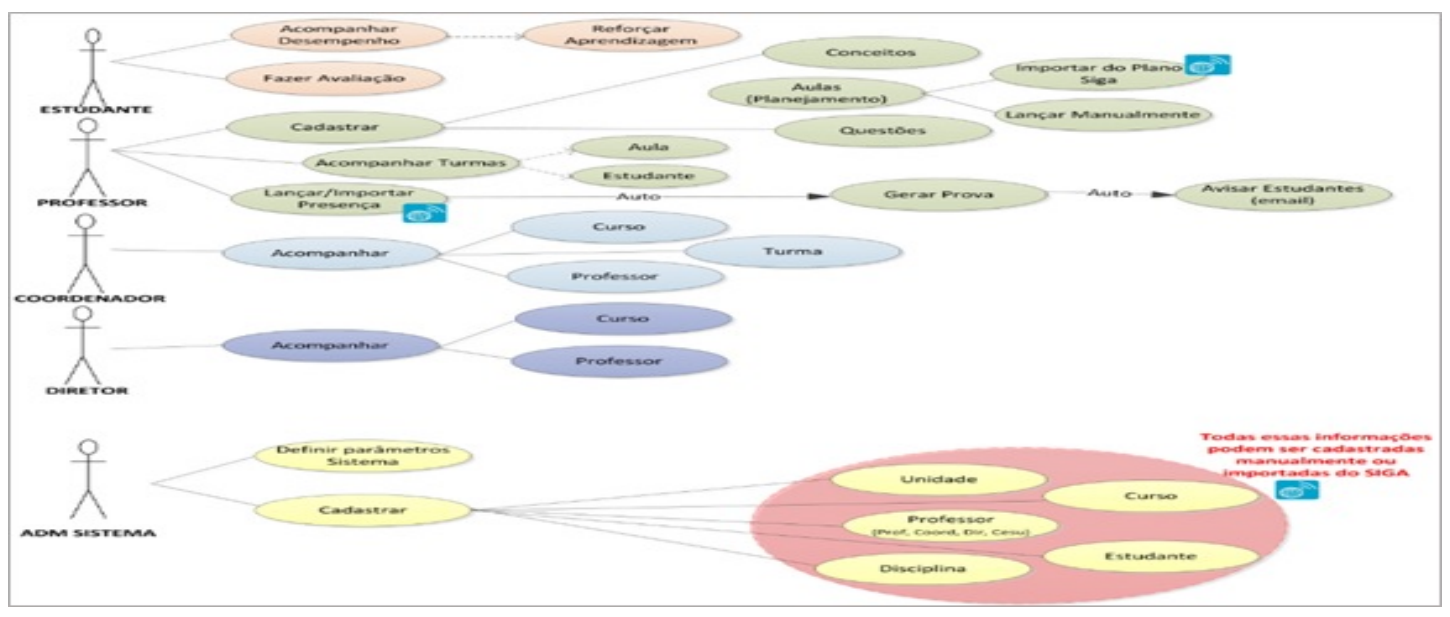

Figura 3 - Modelagem do Sistema SAA 
O que pode ser observado é que, no presente projeto de pesquisa, o foco será a disponibilização das funcionalidades destinadas aos estudantes. Dessa forma, essas funcionalidades estão concentradas na Figura 4, a seguir.

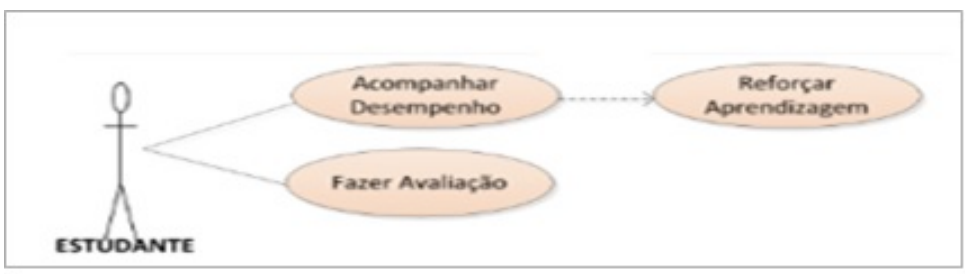

Figura 4 - Processos que devem ser modelados (Funções dos Estudantes no Sistema $S A A)$

\section{Metodologia de desenvolvimento}

Para atingir os objetivos específicos propostos e, por sua vez, o objetivo geral deste projeto, o estudante deverá, primeiramente se concentrar nos estudos sobre a construção de APIs e Webservices, além do ambiente de desenvolvimento de aplicações móveis mais adequado. Uma vez escolhidas as ferramentas, será 0 momento de se concentrar no sistema SAA, entendendo o seu funcionamento, e depois, suas estruturas internas, principalmente os processos e o banco de dados. Só então, 0 estudante terá condições de propor e implementar as alterações necessárias nas estruturas internas do SAA, para prepara-lo e adequá-lo para suportar uma aplicação móvel, baseada em WebServices e APIs. Depois de estruturado o sistema, o estudante deverá partir para o desenvolvimento da aplicação móvel. Ao seu final, deverá testá-la, em casos reais. Por fim, extrair os resultados e construir o relatório científico final.

\section{Resultados esperados}

Ao final do período de doze meses, espera-se que a aplicação móvel para as funcionalidades destinadas aos estudantes do sistema SAA esteja finalizada e devidamente testada.

\section{Considerações finais}

Acredita-se que este projeto será parte importante da pesquisa em desenvolvimento sobre a implementação de metodologias ativas em cursos superiores de tecnologia, possibilitando uma melhor utilização do sistema SAA, o que poderá resultar em melhorias no processo de aprendizagem e, como consequência, uma possível redução nos índices de evasão dos cursos que utilizarem a ferramenta. 


\section{Referencias}

CORRAL, L.; JANES, A.; REMENCIUS, T. Potential Advantages and Disadvantages of Multiplatform Development Frameworks - A Vision on Mobile Environments. Procedia 92 Referências Computer Science, v. 10, p. 1202-1207, jan. 2012. ISSN 1877-0509. Disponível em: .

DRIFTY. lonic: Advanced HTML5 Hybrid Mobile App Framework. 2016. Disponível em:

EL-KASSAS, W. S. et al. Taxonomy of Cross-Platform Mobile Applications Development Approaches. Ain Shams Engineering Journal, 2015. ISSN 2090-4479. Disponível em: .

HOLZINGER, A.; TREITLER, P.; SLANY, W. Making Apps Useable on Multiple Different Mobile Platforms: On Interoperability for Business Application Development on Smartphones. In: QUIRCHMAYR, G. et al. (Ed.). Multidisciplinary Research and Practice for Information Systems. Springer Berlin Heidelberg, 2012, (Lecture Notes in Computer Science, 7465). p. 176-189. ISBN 978-3-642-32497-0 978-3-642-32498-7. DOI: 10.1007/978-3-642-32498-7_14. Disponível em: .

MORAES, D.A.F. (2014). A prova formativa na educação superior: possibilidade de regulação e autorregulação. Estudos de Avaliação Educacional, 25(58), p. 272-294.

PIVA JR., D.; CORTELAZZO, A.L.; FREITAS, F.A. e BELO, R.O. Sistema de Avaliação da Aprendizagem (SAA): Operacionalização da Metodologia Flipped Classroom. In: Anais do 22을 Congresso Internacional ABED Educação a Distância, Águas de Lindóia, SP: ABED, 2016. 